\title{
Classe inversée : quels obstacles en formation des enseignants dans le contexte français?
}

Carole CALISTRI, maître de conférences en Sciences du Langage, Virginie LAPIQUE, docteur en Sciences du Langage, Université de Nice Sophia Antipolis - ESPE de Nice / Laboratoire d'Innovation et Numérique pour l'Education

En ce début de $\mathrm{XXI}^{\mathrm{e}}$ siècle, l'enseignement supérieur doit relever de nombreux défis et permettre la réussite des étudiants, et ces défis se posent avec une acuité particulière dans le domaine de la formation des enseignants français, puisque ces derniers se voient confier la responsabilité d'une classe pour leur année de stage. La maquette de deuxième année de Master en vigueur dans notre université octroie trente heures de formation à la didactique du français. Dans ce contexte, nous avons fait l'hypothèse que la classe inversée offre une perspective intéressante en permettant de « repousser » les limites de l'espace-temps de la formation en présentiel. Plus précisément, notre problématique vise à se demander dans quelle mesure la classe inversée est adaptée au contexte spécifique de la formation des enseignants français, et pourrait favoriser en particulier leur développement professionnel. Des éléments de réponse sont fournis par l'analyse d'entretiens semi-directifs menés avec des étudiants stagiaires, qui permettent d'identifier et de sérier un certain nombre d'obstacles et de freins.

Mots clés : classe inversée - formation des enseignants français - développement professionnalité

\section{Introduction}

En France, la formation initiale des enseignants est partiellement organisée sur le modèle de l'alternance : les étudiants lauréats du concours, associés par l'administration en binômes, se voient attribuer une classe dans laquelle ils exercent alternativement et par périodes de 3 à 4 semaines lorsque l'un des étudiants est en classe, son partenaire est en formation à l'université dans une Ecole Supérieure du Professorat et de l'Education (ESPE) - en revanche, ceux qui n'ont pas réussi le concours effectuent trois stages sous la responsabilité d'enseignants titulaires. II y a donc une différence nette relativement à la responsabilité et au temps de stage entre les deux situations. C'est la première catégorie d'étudiants qui retiendra notre attention dans cette étude.

Les attentes en termes d'efficacité sont élevées : les étudiants reçus au mois de juin enseignent, en pleine responsabilité dès le début du mois de septembre. II faut signaler deux dimensions importantes du 
contexte de formation : les publics formés sont très hétérogènes et le volume horaire dédié à la didactique du français (le domaine d'intervention) représente trente heures dans la maquette de la deuxième année de Master. C'est cette configuration qui a été le déclencheur de la recherche de solutions efficaces, et parmi les possibles, la classe inversée a paru le plus adapté en raison de la capacité qu'elle offre de déployer la formation sur un temps plus important que celui prévu par la maquette (soit 30 heures de travaux dirigés, sans aucun cours magistral).

Dans cet article, l'attention sera focalisée sur l'intérêt que peut représenter la classe inversée en formation des enseignants pour le processus de professionnalisation avec la perspective d'une recherche-action. II sera donc nécessaire de commencer par l'étude de la prescription institutionnelle et de présenter les spécificités des publics d'étudiants concernés.

\section{Éléments de contextualisation}

\section{Prescriptions institutionnelles}

La formation initiale des enseignants est encadrée en France par un référentiel national qui fixe les compétences clés attendues des enseignants: il décline quatorze compétences communes aux professeurs et personnels d'éducation, auxquelles s'ajoutent cinq compétences spécifiques aux seuls professeurs (http://www.education.gouv.fr/cid73215/le-referentiel-de-competences-des-enseignants-aubo-du-25-juillet-2013.html). Le référentiel académique, à l'aune duquel sont évalués les étudiants de Master 2 concernés par notre expérimentation durant leur stage en établissement est quant à lui structuré en six domaines de compétences, qui font écho aux compétences nationales. Le stagiaire doit ainsi :

- agir en fonctionnaire de l'État et de façon éthique et responsable,

- être acteur de la communauté éducative,

- être porteur de savoirs et construire les situations d'enseignement apprentissage,

- mettre en œuvre son enseignement,

- évaluer les acquisitions des élèves et prendre en compte la diversité,

- et enfin démontrer une réflexion et (un) développement professionnel. C'est sur ce dernier axe que portera principalement la réflexion.

En ce qui concerne les modalités de formation, par exemple pour la didactique du français, la maquette de $1^{\mathrm{e}}$ année de master prévoit 74 heures TD réparties sur les deux semestres. Les cours disciplinaires sont largement impactés par la préparation aux épreuves du concours qui intervient en fin d'année. Mais, par ailleurs, pour initier le processus de professionnalisation, les étudiants bénéficient de l'expérience du stage d'observation et de pratique accompagnée de trois semaines et d' "ancrages " (il s'agit d'une séquence de formation qui consiste accompagner les étudiants dans la préparation de séances qui seront mises en œuvre dans des classes puis analysées à leur retour à l'ESPE).

En deuxième année, pour la même unité d'enseignement, le volume horaire prévu descend à trente heures TD avec l'obligation de former les étudiants à l'enseignement de l'oral, de la lecture/écriture, de la littérature et de la langue pour toutes les classes de l'école primaire, donc auprès d'enfants âgés de 3 à 11 ans. Les autres disciplines sont traitées de la même manière. 


\section{Les étudiants du Master 2 « métiers de l'enseignement, de l'éducation et de la formation - parcours premier degré », concernés par le projet}

Ces étudiants sont stagiaires à mi-temps. D'après les fiches renseignées en début d'année, les étudiants ont validé différentes licences (droit, biologie, information et communication, philosophie, Langues Etrangères Appliquées, etc.) ou d'autres masters que le Master "Métiers de l'Enseignement, de l'Education et de la Formation" (économie et gestion, droit et gestion du patrimoine, droit social, industries du tourisme, ingénierie civile, etc.). Une part importante d'entre eux arrive en Master 2 (désormais M2) dans une perspective de reconversion professionnelle, après avoir exercé des métiers très variés : responsable marketing et ressources humaines, secrétaire dans le bâtiment, chef de projet intégrateur informatique, chargé d'études en biologie, hydrobiologiste, ou encore manager dans la location de véhicules, mais aussi quelques cas d'enseignants expérimentés et spécialistes d'une discipline souhaitant changer de niveau d'enseignement. D'autres encore, pères et mères de trois enfants, sont dispensé-e-s de diplômes. Les étudiants ayant suivi le Master 1 «Métiers de l'Enseignement, de l'Education et de la Formation » (désormais MEEF) dans la même ESPE sont donc loin de représenter le seul profil, ni même dans certains groupes le profil majoritaire. L'hétérogénéité est multifactorielle: les étudiants ont / n'ont aucune expérience professionnelle, ont / n'ont aucune expérience professionnelle dans l'enseignement, ont / n'ont pas de diplôme, ont / n'ont pas suivi de cursus ou d'options dans le champ de l'éducation et de la formation, chacune de ces catégories recouvrant une grande diversité de situations.

Ces différents éléments - hétérogénéité des publics, présentiel limité, exigences élevées - ont été déterminants dans notre projet "classe inversée ». En effet, comme le souligne Poumay (2014), les défis que pose l'enseignement supérieur sont nombreux et, dans ce contexte, l'innovation pédagogique paraît incontournable. Questionner le potentiel professionnalisant de la mise en place d'une approche "classe inversée » dans une unité d'enseignement en didactique du français fait partie de nos préoccupations de chercheurs et de formateurs attentifs à l'apprenant en reconfigurant " les espacestemps traditionnels de l'enseigner-apprendre » (Lebrun, 2016, p.15) et, néanmoins, soucieux d'éviter le risque d'une démarche « technocentrée » (Roland, Uyttebrouck, 2015, p.54).

\section{Question de recherche}

La chronologie de notre réflexion est la suivante :

- sériation des contenus disciplinaires et le choix des ressources documentaires et des activités liées. Par exemple, dans le module « enseignement et apprentissage de l'oral », il s'agissait de déterminer les auteurs et travaux incontournables ainsi que les spécificités du domaine (ici la question des difficultés soulevées par l'évaluation), et les dispositifs susceptibles de favoriser l'appropriation des connaissances et la réalisation de séquences d'enseignement efficaces dans leurs classes de stage.

- modalités d'articulation des activités menées à distance et en présentiel.

Ces premiers jalons sont programmatiques et didactiques et avant une mise en œuvre technique et pédagogique coûteuse sur le plan personnel et institutionnel, il était indispensable de répondre à la question de recherche suivante : peut-on identifier à partir de l'analyse des préoccupations des étudiants stagiaires des obstacles dirimants ou susceptibles de freiner notablement la mise en œuvre de la classe inversée en formation des enseignants?

C'est cette recherche et ses résultats qui font l'objet du présent article. 


\title{
Cadres théoriques et méthodologiques
}

\author{
Classe inversée (C.I.) : origines et théories
}

La toute première question légitime est la suivante : quelles sont les origines de la classe inversée? Dans quelle mesure cette approche a-t-elle théorisée?

L'appellation C.I. renvoie historiquement à des expériences récentes menées par des enseignants nordaméricains dans leurs contextes respectifs: en 2007, Bergmann et Sams, enseignants en chimie, popularisent l'utilisation de vidéos visionnées par les élèves en amont de leurs cours. Il s'agit en fait au départ de permettre aux élèves absents d'accéder aux contenus des cours qu'ils auraient ratés, mais en expérimentant ces modalités, les deux enseignants constatent qu'ils sont plus disponibles lors des cours en présentiel pour les questions de leurs élèves, et l'impact sur les interactions leur parait positif. Les «flipped classrooms » sont nées (Bergmann et Sams, 2014). Dans le supérieur, Eric Mazur, professeur de physique appliquée à l'Université d'Harvard, expérimente depuis les années 1990 une méthode interactive baptisée peer instruction (Mazur, 1997), puis conçoit et expérimente un cours qui, conformément à sa devise en anglais - no lectures, no exams - ne comporte ni exposé magistral ni examen, et repose sur le Team Based Learning, un apprentissage collaboratif à partir de projets, articulant enseignement présentiel et à distance. Les principes de son cours, connu sous la dénomination AP50 (Applied Physics 50), sont exposés en ces termes (Dumont et Mazur, 2016, p. 100) :

Le principe de la pédagogie inversée est de modifier de fond en comble l'organisation classique d'un enseignement et de demander aux étudiants de se familiariser avec la théorie avant de venir aux cours (...). Les activités en classe mobilisent les fonctions supérieures d'analyse, de synthèse, d'évaluation et de création selon la taxonomie révisée de Bloom (Anderson, Sosniak, Bloom \& National Society for the Study of Education, 1994).

Cette définition fait écho à celle proposée par le Service de soutien à la formation de l'Université de Sherbrooke sur son site : on peut en effet y lire que cette approche pédagogique,

invers[e] et adapt[e] les activités d'apprentissage traditionnellement proposées aux étudiantes et étudiants en utilisant en alternance la formation à distance et la formation en classe pour prendre avantage des forces de chacune. Dans ce modèle, les contenus de cours sont livrés au moyen de ressources consultables en ligne - le plus souvent des capsules vidéo - et le temps de classe est exclusivement consacré à des projets d'équipe, à des échanges avec l'enseignant et entre pairs, à des exercices pratiques et à d'autres activités de collaboration.

II serait pour autant peut-être imprudent d'affirmer que la C.I. est dotée d'une définition stable et homogène ou qu'elle renvoie à une communauté de pratiques similaires et aisément identifiables. II est certes possible d'identifier des questionnements communs (posture et rôle du formateur, usage du numérique pour optimiser l'enseignement/apprentissage, hybridation, activités en présentiel reposant sur des travaux de groupe et une pédagogie active, rôle et modalités de l'évaluation), mais la diversité des dénominations (méthode, stratégie, modèle, approche, séquence pédagogique) et l'hétérogénéité des pratiques qui se réclament de la C.I. invitent en définitive à la considérer comme "un dispositif pédagogique caractérisé par un ensemble d'intentions et de modalités qui relèvent d'approches, de méthodes, de techniques ou de stratégies déjà existantes » (Nizet, Galiano et Meyer, 2016, p. 43). Peutêtre peut-on considérer le plus petit dénominateur commun en s'appuyant sur l'article d'Héloïse Dufour (2014, p.44) qui indique : 
De manière plus générale cependant, la classe inversée, c'est donner à faire à la maison, en autonomie, les activités de bas niveau cognitif pour privilégier en classe le travail collaboratif et les tâches d'apprentissage de haut niveau cognitif, en mettant les élèves en activité et en collaboration

Une fois posée cette unité et cette diversité de la C.I., prend place la question de la théorisation.

\section{Les fondements théoriques de la C.I.?}

En 2013, Bishop et Verleger entreprennent une revue de la recherche qui vise notamment à faire le point sur les fondements théoriques. Ils soulignent d'abord que les bases mobilisées pour promouvoir la classe inversée se concentrent sur les raisons qui justifient de ne pas utiliser l'enseignement présentiel pour délivrer des cours magistraux. Ces orientations peuvent être associées au constructivisme de Piaget (1967), qui met à l'honneur le conflit cognitif, et au socioconstructivisme de Vygotsky (1978), qui promeut l'apprentissage collaboratif et la prise en compte de la zone proximale de développement. A ces deux courants s'ajoutent la théorie des styles d'apprentissage (Kolb, 1984 ; Felder et Silverman, 1988), l'apprentissage collaboratif, coopératif et assisté par les pairs (Foot et Howe, 1998), basé sur la résolution de problèmes (Barrows, 1986), ou encore la pédagogie active et centrée sur l'apprenant.

En réalité, comme le souligne Tricot, "contrairement aux apprentissages en groupes, où l'idée pédagogique est très étayée scientifiquement, la classe inversée ne dispose pas d'une assise théorique " (Tricot, 2017, p. 100) : elle a en effet été d'abord expérimentée de manière pragmatique ; des fondements pédagogiques lui ont ensuite été associés, mais ils sont divers et non nécessairement compatibles entre eux.

Le contraste entre l'engouement que suscite cette approche et le manque de résultats scientifiques qui permettraient d'asseoir son efficacité sur le plan des apprentissages

La C.I. est en plein essor dans le contexte français : des MOOC lui sont consacrés par Canopé, réseau officiel de création et d'accompagnement pédagogiques dépendant du Ministère de l'Éducation Nationale, l'association Inversons la classe organise des journées d'étude et impulse annuellement des semaines de classe inversée (CLISE), et on peut lire sur le site www.classeinversee.com «Partout dans le monde, des professeurs qui souhaitent offrir la meilleure éducation à leurs élèves passent au modèle de la "classe inversée". Les retours sont unanimes : motivation en hausse, ambiance plus agréable et meilleurs résultats scolaires.»

La C.I. est donc attractive et connait un développement indéniable même si elle suscite par ailleurs des approches critiques (Beitone et Osenda, 2017). Peu d'études scientifiques ont été consacrées à la mesure de son efficacité sur les apprentissages. L'état actuel des recherches n'a ainsi pas permis de démontrer scientifiquement, par le biais de données probantes et d'études contrôlées, que la C.I. permettait d'améliorer significativement l'enseignement et l'apprentissage «Les données probantes associées à la classe inversée sont nettement insuffisantes pour en recommander l'utilisation, particulièrement dans les classes des écoles primaires et secondaires pour lesquelles nous ne disposons actuellement d'aucun résultat de recherche. » (Bissonnette et Gauthier, 2012, p. 26)

En ce qui concerne l'enseignement supérieur, l'étude de Strayer (2012) « qui n'a pas mesuré les effets sur l'apprentissage » ne permettrait pas non plus de justifier scientifiquement la mise en œuvre du dispositif C.I. 


\title{
C.I., développement professionnel des enseignants et théorie de l'activité
}

\author{
Le contexte initial d'expérimentation/création de la classe inversée.
}

Née dans une pratique second degré, mais développée de l'école primaire à l'Université, dans des pays aux formes scolaires culturellement diversifiées, son efficacité et sa pertinence doivent être évaluées en tenant compte des contextes spécifiques dans lesquels elle est mise en œuvre. D'où notre interrogation : la C.I. est-elle adaptée dans un contexte de formation professionnalisante basée sur l'alternance? Les travaux de Nizet et Meyer $(2015,2017)$ fournissent un éclairage sur ce que peut apporter cette approche à la formation initiale de futurs enseignants, mais dans un contexte sensiblement différent du modèle français proposé aux étudiants de deuxième année de Master lauréats du concours auprès desquels nous intervenons. Ainsi, sans sous-estimer l'intérêt d'une réflexion approfondie sur les apports respectifs des activités menées à distance et en présentiel, et donc l'opportunité de (re)penser les apports spécifiques des temps d'activité menés en présence de l'enseignant, de façon à donner le maximum de sens à cette présence (Lebrun, Gilson et Goffinet, 2017; Tricot, 2017), nous avons souhaité adapter notre questionnement aux exigences liées à notre contexte, en interrogeant en premier lieu le potentiel de ce dispositif en terme de développement professionnel.

\section{Obstacles, ressources, développement.}

Nous conduisons notre exploration à l'aide d'outils fournis par la clinique de l'activité, courant d'ergonomie de tradition française initié par $\mathrm{Y}$. Clot et l'équipe de recherche éponyme au CNAM. Une des distinctions importantes de cette psychologie du travail est celle du concept de réel de l'activité (Clot, 1999) :

Le réel de l'activité, c'est aussi ce qui ne se fait pas, ce qu'on ne peut pas faire, ce qu'on cherche à faire sans y parvenir - les échecs - ce qu'on aurait voulu ou pu faire, ce qu'on pense ou qu'on rêve de pouvoir faire ailleurs. II faut y ajouter - paradoxe fréquent - ce qu'on fait pour ne pas faire ce qui est à faire ou qu'on fait sans vouloir le faire. Sans compter ce qui est à refaire.

C'est dire que l'on distingue entre l'activité observable (comportement, occupation) et ce qui, pour n'être pas observable directement, n'en existe pas moins : les pré-occupations, la subjectivité, les sens, les émotions. Ce sont ces éléments - les pré-occupations - que nous nous proposons de repérer et catégoriser et que nous mettrons ensuite en relation avec les cadres de la C.I. tels que nous les avons posés plus haut.

\section{Options méthodologiques}

Nous rappelons qu'il s'agit de l'étape 1 , celle qui permettra de déterminer si les étudiants futurs professeurs des écoles peuvent profiter d'une formation sous le modèle de la C.I.

Les cadres mobilisés pour l'analyse de ces obstacles s'inscrivent dans une démarche qualitative, à visée descriptive, compréhensive et heuristique.

\section{Les données}

II s'agit d'entretiens sur le modèle développé par Vermersch (1994/2015) :

La spécificité de l'entretien d'explicitation est de viser la verbalisation de l'action. (...) Dans toutes les activités qui impliquent des tâches à effectuer (exercices scolaires, activités 
professionnelles, remédiation, analyse de pratique), il est important pour en analyser les difficultés (...) et [les] dysfonctionnement[s], (...) de connaître le déroulement de l'exécution de la tâche. La connaissance du résultat final seul est insuffisante pour diagnostiquer la nature et la cause d'une difficulté ou d'une réussite exceptionnelle. Si par action, je désigne la réalisation d'une tâche, l'entretien d'explicitation vise la description du déroulement de cette action, telle qu'elle a été effectivement mise en œuvre dans une tâche réelle. De plus, ce déroulement d'action est la seule source d'inférences fiables pour mettre en évidence les raisonnements effectivement mis en œuvre (différents de ceux adoptés hors de l'engagement dans l'action), pour identifier les buts réellement poursuivis (souvent distincts de ce que l'on croit poursuivre), pour repérer les savoirs théoriques effectivement utilisés dans la pratique (souvent différents de ceux maîtrisés en question de cours), pour cerner les représentations ou les préconceptions sources de difficultés.

Pourquoi Vermersch? Notre enquête est exploratoire : nous cherchons à savoir si nos étudiants sont en capacité de trouver, sans commande circonscrite, des ressources extérieures et préalables aux TD, donc majoritairement numériques. Cependant, l'absence de commande de la part du formateur ne modifie pas la nécessité, pour les étudiants, d'organiser et mettre en œuvre les enseignements obligatoires dans leurs classes de stage, ce qui réclame une activité de documentation.

Par ailleurs, nous ne nous appuyons ni sur du réalisé (Clot, 1999), en raison du moment de l'année universitaire concerné, ni seulement sur du déclaratif car les discours peuvent receler des représentations (Moscovici, 2003 ; Jodelet, 2003) ou des énoncés marqués par la désirabilité sociale ou professionnelle (Cambon, 2006) : c'est en quoi la perspective de Vermersch est utile puisqu'elle détecte l'action - ce qui est authentiquement engagé chez l'acteur -, en l'occurrence des manifestations d'autonomie dans la recherche des ressources.

\section{Les participants}

La population concerne quatre groupes de professeurs stagiaires, soit 90 étudiants répartis sur deux sites de formation.

\section{La collecte}

Le recueil a eu lieu au mois de septembre dans la première semaine de formation alors que les étudiants (E1 à $\mathrm{E} 4$ dans les verbatims) venaient d'achever une semaine de prise en main de la classe assurée avec leur binôme. II s'agit donc de trois entretiens semi-directifs (un entretien croisé avec deux stagiaires, pour mettre au jour les convergences et les divergences, ainsi que deux entretiens individuels, codés ES1 à ES3),

Parallèlement à ces entretiens, un questionnaire a été renseigné par des étudiants volontaires des quatre groupes (QS1 - QS4). Enfin, des données issues des échanges entre étudiants organisés par le biais de l'application numérique interactive Beekast ont été également recueillies: 118 commentaires sont concernés (APP1 - APP118) ; ils ont été postés au cours du premier module de formation, dans un cours consacré au travail de conception de séquences et de séances pour la classe. En raison de leur volume, ces données seront exploitées en détail dans d'autres publications, mais nous pouvons d'ores et déjà indiquer qu'elles confirment les éléments saillants de l'analyse des entretiens que nous allons exposer maintenant. 


\section{Le guide d'entretien}

Les questions étaient les suivantes:

a. quand vous avez été reçu-e au concours, quelles ont été vos premières réactions ?

b. quand vous avez pensé à votre futur métier, qu'avez-vous envisagé comme ce qui serait le plus facile? le plus difficile?

c. pour préparer votre classe, quelles ont été vos premières actions ?

d. avez-vous déjà rencontré votre binôme?

e. avez-vous des enseignants dans votre famille proche ou dans vos amis?

Le traitement des matériaux

Nous précisons que les transcriptions ont été effectuées comme il est d'usage dans le champ des sciences du langage suivant des conventions répertoriées (ici Vion, 2000), dans une fidélité absolue aux énoncés oraux (qui peuvent donc comporter notamment des phatiques, des troncations, des dislocations, des répétitions...tous éléments signifiants en particulier lorsqu'on recherche, dans le discours, ce qui est obstacle pour l'interlocuteur).

Nous avons donc listé les éléments donnés par les répondants comme pré-occupations (Clot, 1999) et les avons catégorisés en déterminant les tendances et les hapax. Des extraits verbatim seront donnés pour exemplifier nos analyses.

\section{Analyse et discussion des résultats}

L'analyse des données recueillies à travers les entretiens et les questionnaires permet d'identifier quelques axes structurants.

\section{La question des ressources}

Lorsqu'il s'agit de préparer la classe, vers quelles ressources les étudiants se tournent-ils? Lorsque les étudiants sont interrogés sur les actions entreprises, ils accordent une place centrale aux ressources humaines et de proximité.

II s'agit en premier lieu du «binôme», personne avec laquelle ils sont amenés à partager la classe

ES1

78. E2 : moi je me suis tournée vers ma binôme directement

ES2

114.E3 : on a visité l'école ensemble/ on a regardé/ on a fouillé un petit peu dans les placards/'fin voilà/ tout le monde a été de bon conseil/ nous a conseillé des manuels/ 'fin vraiment/ on a bien pris possession des lieux/ déjà/ dès ces premiers jours/ 'fin c'était le dernier jour de l'école quoi/ c'était le huit juillet là je sais plus/ donc voilà/ donc on s'était rencontrés/ puis après on a continué à se voir/ on s'est vus quatre cinq fois au mois de juillet à la maison/ et on a commencé à discuter justement comment on voyait les choses avant de rentrer vraiment voilà dans le vif du sujet/ comment on voyait le fonctionnement de la classe/est-ce qu'on avait le 
même style de pédagogie <rire>/ et après donc on a aussi un peu déblayé au niveau des manuels/ parce que/ alors les commandes avaient été faites quand on est arrivées

mais aussi des étudiants stagiaires de l'année précédente (ES1-E2)

ES1

83. E1: oui elles sont même revenues cette semaine / donc euh / c'est bien de s'entraider comme ça.

En second lieu, les étudiants font référence aux enseignants en poste, soit ceux de l'école où ils sont nommés, soit ceux qui les ont accueillis lors d'une expérience de stage précédente, en première année de master :

ES1

69. E2 : moi ma première action ça a été d'envoyer un mail à notre maitresse de stage parce qu'on avait fait un stage en MS et j'ai une classe PS / MS

70. Ch. : ah

71. E2 : donc j'ai dit / SOS Karine <rires > / aide-moi / donne-moi quelque chose sur quoi me baser pour continuer après parce que : /II

Ces éléments questionnent : alors que la classe inversée accorde une place centrale à la consultation et à l'appropriation de ressources dématérialisées accessibles à distance, ce sont tout d'abord, majoritairement et uniquement vers des personnes que nos étudiants se tournent et, de façon plus paradoxale encore, vers des personnes avec soit peu de métier (leur binôme, les stagiaires de l'année précédente) soit «non labellisés " pourrait-on dire, c'est-à-dire qui ne sont pas des formateurs. La classe inversée, en proposant des ressources, fait, d'une certaine manière, l'hypothèse qu'elles vont rencontrer un besoin. Or, ici, tout se passe comme si les besoins à satisfaire pouvaient l'être sans difficulté, complètement, par le pair ou bien certainement pas dans un espace différent du lieu matériel de la classe. Les étudiants évoquent des inventaires de manuels, de l'organisationnel

\section{ES1}

79 E2. : penser à l'emploi du temps euh / aller dans la classe et sortir les cartons qu'on avait reçus / préparer un peu

QS1 : pour préparer notre classe, ma binôme et moi nous sommes rendues cet été dans l'école et avons tout organisé, trié, et reconditionné

QS3 : j'ai visité ma classe, l'école. (...) J'ai pris des renseignements sur l'école : rythme de l'école (nous sommes sur 4 jours 1/2), nombre d'élèves, liste, horaires (récréations, APC, NAP ...), fournitures commandées pour nous, le matériel présent dans notre classe.

\section{ES3}

144. E4 : on a - -/ et puis comme on ne connaissait pas nos Vagues/ donc on était coincés aussi/ parce qu'on savait pas qui allait commencer donc on a / on avait quand même préparé des fiches de prep pour-euh/ pour notre première semaine/ donc on s'était vraiment réparti des créneaux horaires- - -/ c'était pas forcément les matières/ tout le monde a fait un peu de tout

145. Ch : hummm 
146. E4 : on s'était réparti des créneaux horaires pour que / voilà on ne voulait pas intervenir à deux en même temps/ on s'était laissé genre la première demi-journée ben c'était l'un qui parle et l'autre qui est plutôt en retrait ou en aide aux élèves et inversement/ pour que les enfants quand même comprennent bien qu'on était tous les deux les maîtres/ les maître et maîtresse /

Et l'on pourrait trouver cela étrange : les étudiants dont il est question sont pour deux sur quatre du profil « extérieur » (ils n'ont pas suivi l'année M1 à l'ESPE et ils viennent d'une toute autre branche de métier que celle de l'enseignement). Ils ont la charge pour la première fois de leur vie d'une classe dans laquelle ils vont devoir dispenser les enseignements de dix disciplines...

Les obstacles. Grâce au moment de l'entretien, les étudiants ont pu évoquer la comparaison entre ce qu'ils imaginaient être la part la plus difficile du métier et celle qui l'a réellement été, en tout cas dans les trois premières semaines d'exercice. Aucun élément cité n'est de l'ordre de connaissances préalables ou à compléter ou à initier, c'est-à-dire pouvant être acquises par la consultation.

\section{ES2}

128. E3 : oui et puis voilà/ c'était juste l'inquiétude de se dire voilà/ comment on va faire quoi/ et puis après/ ça aussi on en a discuté/ on connaissait quand même/ on sortait du concours donc/ on avait bien les programmes en tête/

En revanche des transferts de compétence sont envisagés :

\section{ES3}

46. E4 : alors/ euh/ le plus facile 'fin/ alors oui/ je suis revenue/ j'en suis revenue/ mais je pensais très facile que ce serait facile d'obtenir l'attention des élèves

\section{Ch : rire}

48. E4 : d'être calme en classe/parce que j'étais maman et que/bon il n'y avait pas de raison/ je gérais les anniversaires avec quinze élèves donc/ ça se passait bien/ je m'étais dit/ça tout le monde s'en fait un monde/ moi je suis sûre que ça va marcher au pas/bon/ pas du tout/rire voilà ça c'était le truc que je pensais le plus facile/ humm- - - après euh//

Si l'on imaginait de se fier uniquement à ces mentions (ce dont ils disent avoir besoin) pour construire le premier temps de la classe inversée, il paraît bien difficile de penser ici une ressource de type MOOC avec ou sans documentation, des questionnaires, des vidéos de classe... Rien de tout cela n'est LA (vraie) classe que les stagiaires ont en responsabilité... et qui est leur pré-occupation majeure.

Et le numérique ? Bien entendu, nos étudiants l'évoquent, oui, ils vont sur le Net

QS4 : je suis allée sur internet chercher plein d'infos, mais trop d'infos tue l'info! j'étais perdue avec plein de pdf enregistrés dans mon pc sans savoir quoi en faire

et, sans boussole, ils s'y perdent (la question est également de savoir ce qu'ils cherchent vraiment...), et ils utilisent leurs ordinateurs pour communiquer avec leurs binômes !

QS4 : ma binôme m'a expliqué les fiches de préparation, progressions...

\section{ES2}

52 E3. : [...] et on s'est revues pendant l'été/ pour voir où on en était/ on a créé une Dropbox/ pour pouvoir mettre/ tout ce qu'on faisait/ sur la Dropbox et pour pouvoir échanger autour de tout ce qu'on avait fait chacune de notre côté/ voilà/ [...] 


\section{ES3}

48. E4. : alors maintenant/ on s'est abonnées avec mon binôme à un site qui s'appelle Edumoov et qui/ euh/ qui nous permet de concevoir nos fiches de préparation/ tenir notre cahier-journal/ on l'a choisi de manière numérique pour garder plus le lien/ comme ça quand-euh/ même si on s'appelle pas/ on peut jeter un coup d'oeil numériquement/ 'fin sur l'ordinateur/ et on sait ce que l'autre a fait et caetera/ et donc du coup/ c'est vraiment le temps quoi/ c'est que- -/ après je ne sais pas ce qu'on va nous demander exactement/ au niveau du côté du conseiller pédagogique ou du PEMF/ je sais pas trop/[...]

A nouveau, et y compris avec des outils numériques (on remarquera qu'ils sont peu nombreux traitement de texte, messagerie, espace de stockage, une plateforme de service - et pour trois sur quatre courants en dehors du métier d'enseignant), il s'agit de s'adresser à quelqu'un, et les documents ne sont pas cherchés/ trouvés ailleurs que dans l'expérience, ce sont des documents personnels, conçus par les étudiants.

Ces premiers résultats interrogent les préalables à la classe inversée.

Le savoir. Dans les situations de classe inversée, un programme préexiste (comme les notions du cours de physique de Bergmann et Sams) avec une complexité toute relative et sans interférence avec un vécu corrélé. Quelles sont celles de ces dimensions qui se retrouvent dans le cas de la formation des enseignants ? Si des programmes existent, ce sont ceux que nos étudiants auront à enseigner, mais il n'y a pas de parcours simple adapté à la diversité de la réalité des formés (on a pu indiquer la large hétérogénéité des profils d'étudiants) et de la situation de formation, en particulier de la part constituée par le stage en responsabilité.

Le besoin. Cet aspect est neutralisé/naturalisé pourrait-on dire, dans le cadre de la classe inversée, dans le secondaire ou dans le supérieur : dans le secondaire, parce qu'il s'agit de l'obligation scolaire (on ne demande pas aux élèves s'ils ont besoin/ s'ils pensent qu'ils ont besoin d'apprendre la physique, on la leur apprend, ou on tente de le faire), dans le supérieur, parce que, du moins l'espère-t-on, il s'agit d'une orientation, d'un choix disciplinaire. Pour ce qui concerne la formation des enseignants, les besoins que les formateurs identifient (savoir savant, savoir didactique, savoir pédagogique) et ceux que les étudiants identifient (quand ils le font, c'est difficilement et globalement) ne se recouvrent pas. Comment désaltérer quelqu'un qui n'a pas soif/ qui ne se sait pas altéré ?

La quête. La classe inversée postule un ou plusieurs objets d'intérêt pour la communauté formée par le groupe-classe. Dans la formation des enseignants, le nombre des objets possibles est incommensurable, car il est constitué de la spécialité de la situation du stagiaire, ce qu'il désirerait savoir (qui est, pour les novices, davantage ce qu'il désirerait savoir faire) n'est que rarement ce que son camarade désirerait savoir/ savoir-faire. Comment proposer, au préalable, des supports qui tiennent compte des dizaines d'objets - dans le meilleur des cas, dans l'autre, la globalité du désir empêche la sériation - de vingt-cinq étudiants?

Le temps. Dans un système de classe inversée, le présentiel avec l'enseignant (dans le cas qui nous occupe, le formateur) est précédé d'un temps d'apport. La formation en alternance en usage - trois semaines en responsabilité, trois semaines à l'ESPE - placerait donc le temps d'apport (celui où l'étudiant prend contact, connaissance, grâce au truchement de ressources accessibles) pendant la période de stage.

\section{ES3}

70. E4 : donc là je/ j'ai préparé déjà mes trois semaines/ j'ai quasiment il me reste un peu de $\mathrm{X}$ à préparation pour les trois semaines entières à venir lorsque je vais y retourner/ donc là je me dis 
ça va/ je suis sur un coup d'avance/ mais après s'il y a un moment donné / avec je sais pas le mémoire / des devoirs et caetera / je me retrouve la tête dans le guidon / je ne sais pas comment je vais faire / ou alors il faudra en faire moins peut-être je sais pas / donc c'est vraiment le temps que ça prend /

\section{Conclusion}

Le souci qui guide le projet de recherche articulé sur son efficacité dans les faits est celui du développement professionnel. Le référentiel de compétences compte la dimension réflexive comme une sorte de corollaire de ce développement, c'est-à-dire que la temporalité est celle de l'action (à tort ou à raison, le législateur considère que tout le savoir nécessaire est acquis préalablement à l'entrée en formation et que la formation peut donc être ciblée sur les moyens de permettre aux élèves de s'en saisir), sur laquelle le novice peut/ doit revenir. Notre recherche interroge donc les préalables à l'action : peuvent-ils être constitués de ressources communes à un groupe ? au grand groupe des formés ? la dimension pro-active nécessaire est-elle possible dans les cadres actuels ? quelles habitudes d'autonomie/ de circonscription de leurs besoins les futurs professeurs doivent-ils posséder ? quelle familiarité avec les objets numériques?

C'est peut-être avec l'inclusion dans un ensemble plus vaste, comme par exemple un continuum depuis la première année de licence, ainsi qu'une exploration du mentorat médié que ces questions pourraient trouver des éléments de réponse.

\section{Bibliographie}

BEITONE A., OSENDA M. (2017), «La pédagogie inversée: une pédagogie archaïque», in Skhole.fr : http://skhole.fr/lapedagogie-inversee-une-pedagogie-archaique-par-alain-beitone-et-margaux-osenda (consulté le 11/03/2018).

BERGMAN J., SAMS A. (2012), Flip your classroom : Reach every student in every class every day. Eugene, OR \& Washington DC : International Society for Technology in Education.

BISHOP J.-L., VERLEGER M.-A. (2013), "The flipped classroom: A survey of the research", in ASEE National Conference proceedings, Atlanta, GA (30), p. 1-18.

BISSONNETTE S., GAUTIER C. (2012), « Faire la classe à l'endroit ou à l'envers? Traditional or flip teaching », in Formation et profession, 20/1, p. 23-28.

CAILLIEZ J.-C. (2017), La classe renversée. L'innovation pédagogique par le changement de posture, Paris : Ellipses.

CAMBON L. (2006), « Désirabilité sociale et utilité sociale, deux dimensions de la valeur communiquée par les adjectifs de personnalité ». Revue internationale de psychologie sociale, tome 19,(3), p.125-151

CLOT Y. (1999), La fonction psychologique du travail, Paris : PUF.

DUFOUR, H.(2014), « La classe inversée », Revue Technologie 193, p.44.

DUMONT A., Mazur E. (2017), « Une pratique éprouvée de la classe inversée pour l'enseignement à la Harvard University, aux Etats-Unis », in A. Dumont \& D. Berthiaume, La pédagogie inversée. Enseigner autrement dans le supérieur avec la classe inversée, Bruxelles : De Boeck, p. 95-114.

LEBRUN M. (2016), « La classe inversée au confluent de différentes tendances dans un contexte mouvant » in A. Dumont \& D. Berthiaume, La pédagogie inversée. Enseigner autrement dans le supérieur avec la classe inversée, Bruxelles : De Boeck, p. 13-38.

LEBRUN M., GILSON C. \& GOFFINET C. (2017), « Contribution à une typologie des classes inversées : éléments descriptifs de différentes types, configurations pédagogiques et effets », in Education \& Formation, e-306, p.125-145.

MAZUR E. (1997), Peer instruction : a user's manual, Upper Saddle River, NJ : Prentice Hall. 
MOSCOVICI S. (2003) «2. Des représentations collectives aux représentations sociales : éléments pour une histoire », in D. Jodelet, Les représentations sociales, Paris : Presses Universitaires de France. p. 79-103.

NIZET I., MEYER F. (2015), « La classe inversée : que peut-elle apporter aux enseignants ? ", L'Agence des usages des TICE, Paris : Canopé.

NIZET I., MEYER F. (2017), « Une expérience de classe inversée en formation initiale de futurs enseignants », in A. Dumont \& D. Berthiaume, La pédagogie inversée. Enseigner autrement dans le supérieur avec la classe inversée, p. $163-176$.

POUMAY M. (2014), « Six leviers pour améliorer l'apprentissage des étudiants du supérieur », in Revue internationale de pédagogie de l'enseignement supérieur, $\mathrm{n}^{\circ} 30-1$.

REGE COLET N., MCALPINE L., FANGHANEL J. \& WESTON C. (2011), « Le concept de Scholarship of Teaching and Learning », Recherche et formation, $n^{\circ} 67$, p. 91-104.

STRAYER J.-F. (2012), « How learning in an inverted classroom influences cooperation, innovation and task orientation », in Learning Environments Research, 15(2), p. 171-193.

TRICOT A. (2017), L'innovation pédagogique. Mythes et réalités, Paris : Retz.

VERMERSCH P. (1994), L'entretien d'explicitation en formation initiale et continue, Paris : ESF.

VION R. (2000), La communication verbale. Analyse des interactions, Paris : Hachette. 